\title{
Progressive disseminated histoplasmosis with concomitant disseminated nontuberculous mycobacterial infection in a patient with AIDS from a nonendemic region (California)
}

\author{
Julian Choi ${ }^{1}$, Kia Nikoomanesh ${ }^{2 *}$, Jusleen Uppal ${ }^{2}$ and Sharon Wang ${ }^{3}$
}

\begin{abstract}
Background: Opportunistic infections, while well studied in the AIDS population, continue to have variable and surprising presentations. Here, we present a case of disseminated histoplasmosis with disseminated nontuberculous mycobacterial infection in a 50 year old man with long standing AIDS living in a non-endemic area.

Case presentation: Patient presented with a constellation of symptoms, and imaging of the chest showed a pulmonary mass with cavitation, multiple nodules, and ground glass opacities. Further investigations revealed granulomatous lung nodules and fungemia consistent with Histoplasma capsulatum, and coinfection with disseminated nontuberculous mycobateria in a nonendemic area.

Conclusions: Immunocompromised patients risk co-inhabitation by multiple infectious organisms. Some of these organisms may preside in the host for years prior to reactivation. Clinicians in non endemic areas should therefore be careful to not overlook specific organisms based on a lack of a recent travel history. Physicians in nonendemic areas should become more familiar with the clinical findings and diagnostic approach of infectious such as Histoplasmosis, to ensure earlier recognition and treatment in immunocompromised individuals.
\end{abstract}

Keywords: Histoplasmosis, AIDS, Nontuberculous mycobacterial infection, Nonendemic, Opportunistic infections, Reactivation, California, Co-infection

\section{Background}

Histoplasma capsulatum is an endemic, dimorphic fungus commonly found in the midwest and southern United States (US), and most prevalent along the Ohio and Mississippi river valleys. It is the most common cause for hospitalization of the endemic mycoses [1]. Patients with acquired immune deficiency syndrome (AIDS) have weakened cellular immunity and increased risk for histoplasmosis especially in areas of endemic foci. However, in nonendemic areas such as California (where our patient resides), it is thought to be due to reactivation of latent

\footnotetext{
* Correspondence: kianikoomanesh@gmail.com

${ }^{2}$ Department of Internal Medicine, Arrowhead Regional Medical Center,

Colton, USA

Full list of author information is available at the end of the article
}

infection from previous exposure rather than an acute infection [2-5]. Clinical presentation depends on the immune status of the host and extent of exposed inoculum. Healthy individuals are typically asymptomatic or have self-limiting infections, while many with AIDS present with progressive disseminated histoplasmosis with pulmonary involvement $[1,4,6]$. Herein we present a patient with AIDS coinfected with disseminated nontuberculous mycobacterial infection and progressive disseminated histoplasmosis in southern California.

\section{Case presentation}

A 50-year-old homeless Caucasian man with history of AIDS presented for generalized weakness and productive cough with clear-yellow sputum without hemoptysis for 
1 month. He also endorsed fevers, chills and rigors for 1 week and a 15 pound unintentional weight loss in 1 month. AIDS was diagnosed over 20 years ago and has been noncompliant with various combinations antiretroviral therapy (cART) regimens including emtricitabine/ tenofovir, abacavir/lamivudine, darunavir, and ritonavir. Patient was lost to follow-up for 2 years until he was recently incarcerated and released from jail. Patient was born in Ohio but moved to California at 2 years of age, and had remote military service in Georgia in his early 20's. Otherwise the patient never left California thereafter. He has never explored caves or been in contact with birds, bats or its excrements.

Patient's initial temperature was $38.5^{\circ}$ Celsius and he was also tachycardic. Physical exam revealed a disheveled, cachectic male with temporal muscle wasting, no respiratory distress on room air, and was otherwise unremarkable.

Laboratories revealed a white blood cell count of 3.7 $\mathrm{TH} / \mathrm{uL}$, absolute lymphocyte count of 185 , absolute CD4 count of 20 cells/uL, and HIV viral load of 181,000 copies $/ \mathrm{mL}$. Comprehensive metabolic panel was within normal ranges except for a low albumin $(2.8 \mathrm{~g} / \mathrm{dL})$. Lactate dehydrogenase $(277 \mathrm{u} / \mathrm{L})$, ferritin $(1343 \mathrm{ng} / \mathrm{mL})$, erythrocyte sedimentation rate $(111 \mathrm{~mm} / \mathrm{hr})$, and C-reactive protein $(9.58 \mathrm{mg} / \mathrm{dL})$ were elevated. Computed tomography (CT) of the chest with contrast revealed bilateral nodular opacities, the largest measured $(3.6 \times 2.2 \mathrm{~cm})$, a left upper lobe mass with cavitation, right basilar $(2.0 \times 1.5 \mathrm{~cm})$ nodule, mediastinal lymphadenopathy, ground-glass changes, and enlarged periaortic lymph nodes (Fig. 1a). CT abdomen and pelvis revealed retroperitoneal lymphadenopathy and was otherwise unremarkable.

The initial workup included a lumbar puncture which was unremarkable. Multiple sputum acid fast bacilli (AFB) smears were negative. Interestingly, serum and urine were positive for rare AFBs using fluorochrome staining but nucleic acid amplification testing (NAAT) for Mycobacterium tuberculosis complex were negative, raising suspicion for disseminated nontuberculous mycobacterial (NTM) infection. Patient was started on treatment for presumed disseminated NTM infection with daily regimen of azithromycin/ rifabutin/ethambutol with resolution of fever. However, patient still endorsed a cough. He subsequently underwent image guided fine needle aspiration of peripheral lung

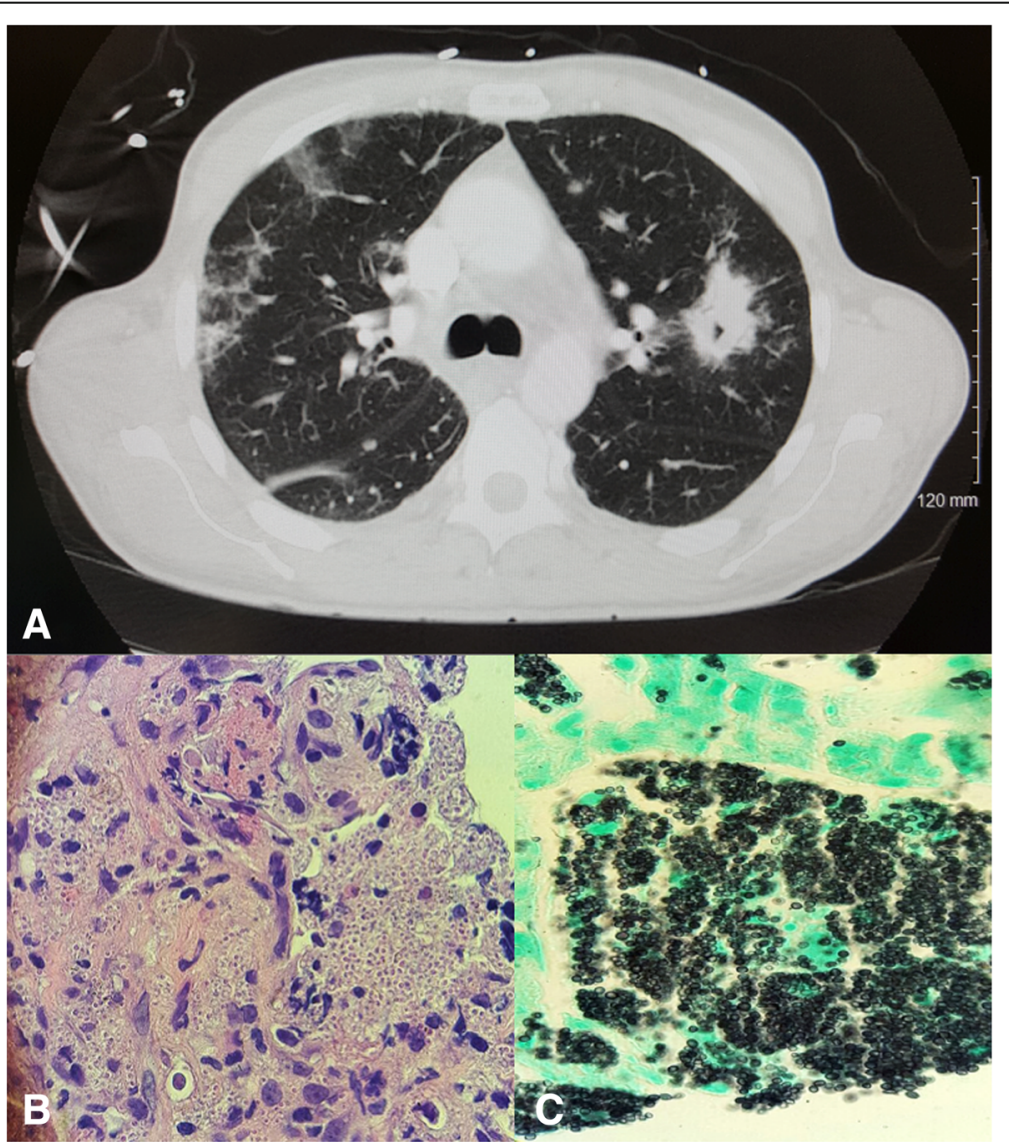

Fig. 1 a CT chest showing left lung cavitary mass, nodular and ground-glass opacities, and mediastinal lymphadenopathy. b Hematoxylin and eosin (H\&E) stain showing granuloma formation and dimorphic yeast. c Gomori Methenamine silver (GMS) stain showing numerous dimorphic oval-shaped budding yeast consistent with Histoplasma capsulatum 
nodules. Pathology (Fig. 1b \& c) revealed granulomatous inflammation with abundant Histoplasma capsulatum organisms by Grocott-Gomori's methenamine silver stain (GMS) staining. There were no AFB positive organisms seen. Of note, the diagnosis was further supported by positive urine Histoplasma antigen $(>19 \mathrm{ng} / \mathrm{ml}$ ) and serum Histoplasma antigen above the limit of quantification. Histoplasma antibody with mycelial antigen was $<1: 8$. Blood and final bronchoscopy cultures eventually grew $H$. capsulatum weeks later.

Once diagnosis of $H$. capsulatum was made, in addition to NTM treatment regimen, the patient was also started on intravenous amphotericin B lipid suspension at $5 \mathrm{mg} / \mathrm{kg}$ for 2 weeks and subsequently discharged with itraconazole. Patient was also started on cART (emtricitabine/tenofovir/ dolutegravir) prior to discharge. With treatment, all constitutional symptoms and laboratory abnormalities improved. He was subsequently discharged home with close outpatient follow-up.

\section{Conclusion}

It is well known that Histoplasma capsulatum is endemic to the Ohio and Mississippi river valley regions of the US. According to articles from the Centers for Disease Control and Prevention (CDC), between the years 1938 and 2013, there were 2850 reported cases of histoplasmosis spread out across 26 states of the US [7]. Of those cases, none were found in California. This seems to be similar for the years 2011-2014, with 3409 reported cases in 12 states, none being in the west coast [8]. Although the CDC does not list $H$. capsulatum to be a reportable fungal disease in California, one could presume that identifying histoplasmosis in California (and other nonendemic states) is rare, or, underdiagnosed and underreported.

In the era of cART therapy HIV infected individuals have the opportunity to live longer and travel around the globe, potentially increasing the opportunity of making a diagnosis of histoplasmosis in nonendemic areas [9]. Histoplasmosis is a common opportunistic infection in endemic regions for individuals with AIDS. They are at higher risk for histoplasmosis with up to $25 \%$ occurrence in select cities compared to $1 \%$ in nonendemic areas $[6,10]$.

Clinical presentation of histoplasmosis depends on the volume of exposure, host immune status, and overall health. Histoplasmosis can be the first manifestation of AIDS in up to $50-75 \%$ of patients with disseminated infection with mortality rates as high as $39-58 \%$ depending on endemicity [9]. Traditionally, progressive disseminated histoplasmosis (PDH) presents with myriad of non-specific symptoms and examination findings: fevers, night sweats, weight loss, cough, dyspnea, fatigue, hepatosplenomegaly, lymphadenopathy, and less frequently, skin, gastrointestinal, and central nervous system manifestations $[1,4,6]$. PDH is usually seen in AIDS patients with absolute CD4 counts of less than 150-200 cells/uL $[9,11]$. Other associated laboratory values (although non-specific) include: pancytopenia, elevated ferritin, lactate dehydrogenase, and abnormal liver function tests $[1-3,12]$.

Histoplasmosis diagnosed in nonendemic regions, as seen with this particular case, was likely from reactivation of latent infection in those without recent exposure $[2,3,5,10,13]$. Our patient likely was exposed in Ohio during his first 2 years of life, or in Georgia prior to traveling to southern California. His detailed travel history accounted for no other visits to endemic areas for over 20 years. He was also diagnosed with HIV for 20 years and was compliant in the past with antiretroviral therapy until about 2 years from presentation.

There is high variability between time of travel to endemic areas and manifestations of clinical symptoms. One survey in Europe described symptoms occurring between 2 months to 5 years after travel to endemic area [14]. They also describe individuals in the United Kingdom who served in World War II in endemic areas who had latency periods more than 50 years before reactivation. It is prudent that a detailed travel history be obtained to elicit past exposures from endemic areas to not delay recognition and treatment for histoplasmosis. During that time he likely had declining cellular immunity and endogenous reinfection from weakened host defenses. Furthermore, there are reports of certain biologic medications being associated with reactivation occurring in nonendemic regions that may be related to decreased immunity [5].

Clinicians in nonendemic areas may not consider histoplasmosis as a diagnosis especially if patients have co-infections that could potentially account for clinical and laboratory irregularities. However, multiple concurrent infections are very common in patients with AIDS. $[6,13,15,16]$. Fredericks et. al. reviewed 46 patients from two San Francisco hospitals with disseminated histoplasmosis and AIDS. 54\% had coinfections and only 1 of the cases listed histoplasmosis as part of their original differential diagnosis [3]. Kitkungvan et al. performed an observational study in a Thailand hospital showing $26 \%$ of their HIV infected individuals presenting with fever of unknown origin to have co-infections with 2 or more pathogens [17]. The principle of "Occam's razor" is that the simplest solution is often correct. However, in patients with AIDS, this can misguide investigators in making erroneous assumptions. Instead, it may better serve clinicians caring for these immunocompromised individuals to apply the concept of "Hickam's dictum." It is a paradigm in which a patient's manifestations are better explained not by a single unifying diagnosis, but multiple diseases processes occurring at once. In the era of cART and increased global travel, endemicity of a particular infectious 
disease may no longer be localized (as in our case) to a specific region. Physicians in nonendemic areas should become more familiar with the clinical findings and diagnostic approach of histoplasmosis, to ensure earlier recognition and treatment for this vulnerable group of individuals.

\section{Abbreviations}

AFB: Acid Fast Bacilli; AIDS: Acquired immune deficiency syndrome; CART: Combinations antiretroviral therapy; CD4: Cluster of differentiation 4; CDC: Center for Disease Control and Prevention; Cells/uL: Cells per microliter; cm: Centimeter; g/dl: Grams per deciliter; mg/dl: Milligrams per deciliter; Mg/ kg: Milligram per kilogram; mm/hr.: Millimeters per hour; NAAT: Nucleic acid amplification test; ng/ml: Nanogram per milliliter; NTM: Nontuberculous mycobacterium; PDH: Progressive disseminated Histoplasmosis; u/L: Units per

\section{Acknowledgements}

Not applicable.

\section{Funding}

No funding was involved in this case study.

\section{Availability of data and materials}

Not applicable.

\section{Authors' contributions}

$J C, K N, J U$, and SW all aided in the formulation and the direction of the manuscript. All members were involved in writing the draft. All members worked on editing the draft after completion and worked to alter the manuscript into its final form. All authors have read, and approved the final draft of this manuscript.

\section{Ethics approval and consent to participate}

Not applicable.

\section{Consent for publication}

We attest that informed consent for publication of identifying information/ imaging in an online publication was attained. Informed consent was obtained in writing.

\section{Competing interests}

The authors declare that they have no competing interests.

\section{Publisher's Note}

Springer Nature remains neutral with regard to jurisdictional claims in published maps and institutional affiliations.

\section{Author details}

'Department of Pulmonary and Critical Care Medicine, Arrowhead Regional Medical Center, Colton, USA. ${ }^{2}$ Department of Internal Medicine, Arrowhead Regional Medical Center, Colton, USA. ${ }^{3}$ Department of Infectious Disease, Arrowhead Regional Medical Center, Colton, USA

Received: 26 September 2018 Accepted: 8 February 2019

Published online: 21 February 2019

\section{References}

1. Kauffman CA, Marr KA, Mitty J. Pathogenesis and clinical features of pulmonary histoplasmosis, UpToDate. Waltham, MA: UpToDate Inc:; 2017. http://www.uptodate.com

2. Davies SF, Khan M, Sarosi GA. Disseminated histoplasmosis in immunologically suppressed patients, occurrence in a nonendemic area. Am J Med. 1978;64:94-100

3. Fredricks DN, Rojanasthien N, Jacobson MA. AIDS-related disseminated histoplasmosis in San Francisco, California. West J Med. 1997;167:315-21.

4. Wheat J. Histoplasmosis: a review for clinicians from non-endemic areas. Mycoses. 2006;49(4):274-82.
5. Jain V, Evans T, Peterson MW. Reactivation histoplasmosis after treatment with anti-tumor necrosis factor a in a patient from a nonendemic area. Respir Med. 2006;100:1291-3.

6. Wheat $L$, Connolly-Stringfield PA, Baker RL, Curfman MF, Eads ME, Israel KS, Norris SA, Webb DH, Zeckel ML. Disseminated histoplasmosis in the acquired immune deficiency syndrome: clinical findings, diagnosis and treatment, and review of the literature. Medicine (Baltimore). 1990;69(6): 361-74.

7. Benedict K, Mody RK. Epidemiology of histoplasmosis outbreaks, United States, 1938-2013. Emerg Infect Dis. 2016;22(3):370-8. https://doi.org/10. 3201/eid2203.151117.

8. Armstrong PA, Jackson BR, Haselow D, et al. Multistate epidemiology of histoplasmosis, United States, 2011-20141. Emerg Infect Dis. 2018;24(3):42531. https://doi.org/10.3201/eid2403.171258.

9. Adenis AA, Aznar C, Couppié P. Histoplasmosis in HIV-infected patients: a review of new developments and remaining gaps. Cur Trop Med Rep. 2014; 1(2):119-28. https://doi.org/10.1007/s40475-014-0017-8.

10. Wheat J. Endemic mycoses in AIDS: a clinical review. Clin Microbiol Rev. 1995:8(1):146-59.

11. Mandell LA, Wunderink RG, Anzueto A, Bartlett JG, Campbell GD, Dean NC, Dowell SF, File TM Jr, Musher DM, Niederman MS, Torres A, Whitney CG. infectious Diseases Society of America/American Thoracic Society consensus guidelines on the management of community-acquired pneumonia in adults. Clin Infect Dis. 2007:44(Suppl 2):S27-72.

12. Howard DH. Acquisition, transport, and storage of Iron by pathogenic fungi. Clin Microbiol Rev. 1999:12(3):394-404.

13. Antinori S, Magni C, Nebuloni M, Parravicini C, Corbellino M, Sollima S, et al. Histoplasmosis Among Human Immunodeficiency Virus-Infected People in Europe. Medicine (Baltimore). 2006;85:22-36 Doil.

14. Ashbee HR, Evans EG, Viviani MA, Dupont B, Chryssantho E, Surmont I, Tomsikova A, Vachkov P, Energo B, Zala J, Tintelnot K. Histoplasmosis in Europe: a report on an epidemiological survey from the European Confederation of Medical Mycology Working Group. Med Mycol. 2008;46(1):57-65.

15. Agudelo CA, Restrepo CA, Molina DA, et al. Tuberculosis and histoplasmosis co-infection in AIDS patients. Am J Trop Med Hyg. 2012;87(6):1094-8. https://doi.org/10.4269/ajtmh.2012.12-0292.

16. Taján J. Espasa M, Sala M, et al. Disseminated infection by Mycobacterium sherrisii and Histoplasma capsulatum in an African HIV-infected patient. Am J Trop Med Hyg. 2013;88(5):914-7. https://doi.org/10.4269/ajtmh.12-0572.

17. Kitkungvan D, Apisarnthanarak A, Plengpart P, Mundy LM. Fever of unknown origin in patients with HIV infection in Thailand: an observational study and review of the literature. Int J STDA \& AIDS. 2008;19(4):232-5.

\section{Ready to submit your research? Choose BMC and benefit from:}

- fast, convenient online submission

- thorough peer review by experienced researchers in your field

- rapid publication on acceptance

- support for research data, including large and complex data types

- gold Open Access which fosters wider collaboration and increased citations

- maximum visibility for your research: over $100 \mathrm{M}$ website views per year

At BMC, research is always in progress.

Learn more biomedcentral.com/submissions 